\title{
Generalization of Szász operators: quantitative estimate and bounded variation
}

\author{
Bozkurt K. ${ }^{1}$, Limmam M.L. ${ }^{2}$, Aral A. ${ }^{2,}$, \\ Difference of exponential type Szász and Szász-Kantorovich operators is obtained. Similar es- \\ timates are given for higher order $\mu$-derivatives of the Szász operators and the Szász-Kantorovich \\ type operators acting on the same order $\mu$-derivative of the function. These differences are given in \\ quantitative form using the first modulus of continuity. Convergence in variation of the operators in \\ the space of functions with bounded variation with respect to the variation seminorm is obtained. \\ The results propose a general framework covering the results provided by previous literature. \\ Key words and phrases: exponential Szász operator, exponential Szász-Kantorovich operator, con- \\ vergence in variation.

\footnotetext{
${ }^{1}$ National Defense University, Turkish Military Academy, Devlet Mah. Kara Harp Okulu Cd., 06420 Çankaya, Ankara, Turkey

${ }^{2}$ Kırıkkale University, Ankara Cd., 71450 Yahşihan, Kırıkkale, Turkey

$\triangle$ Corresponding author

E-mail: kenanbozkurt06@gmail.com (Bozkurt K.), mdlimam90@gmail.com (Limmam M.L.), aliaral73@yahoo.com (Aral A.)
}

\section{Introduction}

In recent years, the investigations of differences of two linear positive operators have attracted the attention of scientists. The reason it is still a popular research area is that such differences have some information about the approximation properties of the operators given to us. This means that if the approximation properties of one of the operators are known, similar properties of the other can be determined. With this viewpoint, A. Lupaş [21] obtained quantitative type theorems for the difference of $B_{n} \circ \overline{\mathbb{B}}_{n}-\overline{\mathbb{B}}_{n} \circ B_{n}$, where $B_{n}$ and $\overline{\mathbb{B}}_{n}$ are Bernstein and Beta operators, respectively. After this fundamental study, H. Gonska and I. Raşa [15] obtained more general results in regard to Lupaş' problem using the Taylor expansion with Peano remainder in view of the least concave majorant of the modulus of continuity. H. Gonska, P. Pitul and I. Raşa in [14] have continued their research on the differences of positive linear operators by giving estimates about the Bernstein operators, and Beta operators of the second kind, as introduced by Lupaş. In [16], similar results were presented with emphasis on the use of the second modulus of continuity. A.M. Acu and I. Raşa in [5] obtained new estimates of the differences of certain positive linear operators using Taylor's formula. In [6], the quantitative estimates for the differences of certain positive linear operators and their derivatives, provided some numerical results. Very recently, A. Aral and H. Erbay in [9] presented the estimate of the difference of the exponential-type Bernstein and Bernstein-Kantorovich operators. Also, they studied the difference of mentioned operators and their $\mu$-derivatives with supporting the theory with some numerical results. Other contributions can be found in [8] 
and [10]. Because of better approximation properties of the operators preserving exponential operator than classical ones, similar contributions have been the subject of research in other well-known operators. In this sense, we recall the sequence $\mathcal{S}_{n}$ of linear positive operators introduced and studied in [2] and [3], that are called exponential Szàsz operators. The operators preserving the exponential functions $\exp (\mu t)$ and $\exp (2 \mu t), \mu>0,\left(\exp _{\mu}(x)=e^{\mu x}\right)$ are defined by

$$
\mathcal{S}_{n} f(x)=\mathcal{S}_{n}(f ; x)=e^{-n \alpha_{n}(x)} \sum_{k=0}^{\infty} f\left(\frac{k}{n}\right) e^{-\mu k / n} e^{\mu x} \frac{\left(n \alpha_{n}(x)\right)^{k}}{k !},
$$

where $x \in \mathbb{R}^{+}, n \in \mathbb{N}$,

$$
\begin{gathered}
\alpha_{n}(x)=\frac{\mu x}{n\left(e^{\mu / n}-1\right)} \\
\mathcal{S}_{n} f(x)=\exp _{\mu}(x) S_{n}\left(\frac{f}{\exp _{\mu}} ; \alpha_{n}(x)\right),
\end{gathered}
$$

where $S_{n}(\cdot ; x)$ is the classical Szàsz operators for a fixed real parameter $\mu>0$. As usual, we denote by $e_{i}$ the polynomial functions defined by $e_{i}(t)=t^{i}$.

In order to furnish an approximation process for space of integrable functions on the interval $[0, \infty)$, integral modification of the operators $\mathcal{S}_{n}$ are defined in [11] by setting for $n \geq 1$, $\mu \geq 0$ and $x \in \mathbb{R}^{+}$

$$
\mathcal{K}_{n}=D_{\mu} \circ \mathcal{S}_{n} \circ I_{\mu}
$$

where the operators $D_{\mu}: C^{1}\left(\mathbb{R}^{+}\right) \rightarrow C\left(\mathbb{R}^{+}\right)$and $I_{\mu}: C\left(\mathbb{R}^{+}\right) \rightarrow C^{1}\left(\mathbb{R}^{+}\right)$, and defined by

$$
\begin{gathered}
I_{\mu}(f, x)=e^{\mu x} \int_{0}^{x} e^{-\mu t} f(t) d t, \quad f \in C\left(\mathbb{R}^{+}\right) \text {and } x \in \mathbb{R}^{+}, \\
D_{\mu}(f, x)=f^{\prime}(x)-\mu f(x) \quad f \in C^{1}\left(\mathbb{R}^{+}\right) \text {and } x \in \mathbb{R}^{+},
\end{gathered}
$$

(see [22]). Using the operators in (3), which are different from the classical approach, we have

$$
\mathcal{K}_{n} f(x)=n \beta_{n} e^{-n \alpha_{n}(x)} e^{\mu x} \sum_{k=0}^{\infty} \frac{\left(n \alpha_{n}(x)\right)^{k}}{k !} \int_{k / n}^{(k+1) / n} f_{\mu}(t) d t,
$$

where $\alpha_{n}(x)$ is given in (2), $\alpha_{n}^{\prime}(x)=\beta_{n}$ and $f_{\mu}(t)=e^{-\mu t} f(t)$. Other contributions on this topic can be found in $[17,18]$ and recently [12].

The present article consists of two parts. In the first part, some auxiliary conclusions regarding higher order $\mu$-derivative of the operators $\mathcal{S}_{n}$ and $\mathcal{K}_{n}$ are obtained. Then, we prove some estimates of the differences of the modified exponential-type Szàsz operator and SzàszKantorovich variant and their differences between their higher order $\mu$-derivatives of the operators with the operators applied to the same order of $\mu$-derivative of the function, respectively. Also, a relation between the operators is presented.

In the second part, another outstanding tool to achieve the measurement of the effectiveness of the approximation, variation detracting property is introduced. Although this topic was first studied by G.G. Lorentz [20], important contributions were given in [13] which presented that both Bernstein and genuine Kantorovich operators possess the variation detracting property. Very recently, in [4] the variation detracting property of Bernstein-Kantorovich operators was presented. For the operators defined on unbounded interval similar contributions can be found in $[1,7,19]$. Based on the articles, in order to obtain a convergence result in the variation seminorm, firstly we state the variation detracting property for the operators $\mathcal{S}_{n}$ and $\mathcal{K}_{n}$. 


\section{Auxiliary results}

Firstly we recall exponential moments of the operator $\mathcal{S}_{n}$ and $\mathcal{K}_{n}$.

Lemma 1. For the operators $\left(\mathcal{S}_{n}\right)_{n \geq 1}$ we have:

1) $\mathcal{S}_{n}\left(e_{0} ; x\right)=e^{\mu x\left(1-e^{-\mu / n}\right)}$;

2) $\mathcal{S}_{n}\left(\exp _{\mu} ; x\right)=e^{\mu x}$;

3) $\mathcal{S}_{n}\left(\exp _{\mu}^{2} ; x\right)=e^{2 \mu x}$.

Lemma 2. For the operators $\left(\mathcal{K}_{n}\right)_{n \geq 1}$ we have:

1) $\mathcal{K}_{n}\left(e_{0} ; x\right)=e^{\mu x\left(1-e^{-\mu / n}\right)-m / n}$;

2) $\mathcal{K}_{n}\left(\exp _{\mu} ; x\right)=\mu e^{\mu x} /\left(n\left(e^{\mu / n}-1\right)\right)$;

3) $\mathcal{K}_{n}\left(\exp _{\mu}^{2} ; x\right)=e^{2 \mu x}$.

Let us calculate the $r$-th order $\mu$-derivative of the operator $\mathcal{S}_{n}$.

Lemma 3. For every $r \in \mathbb{N}$ and $n \in \mathbb{N}$, we have

$$
D_{\mu}^{r} \mathcal{S}_{n}(f ; x)=n^{r} \beta_{n}^{r} \mathcal{S}_{n}\left(\exp _{\mu} \Delta_{1 / n}^{r} f_{\mu} ; x\right), \quad x \in \mathbb{R}^{+},
$$

where

$$
\Delta_{h}^{r} f(x)=\sum_{k=0}^{r}\left(\begin{array}{l}
r \\
k
\end{array}\right)(-1)^{r-k} f(x+k h) .
$$

Proof. Using the formula (4), it is easily seen that

$$
\begin{aligned}
D_{\mu} \mathcal{S}_{n}(f ; x)= & \mu e^{-n \alpha_{n}(x)} e^{\mu x} \sum_{k=0}^{\infty} \frac{\left(n \alpha_{n}(x)\right)^{k}}{k !} f_{\mu}\left(\frac{k}{n}\right)+n \alpha_{n}^{\prime}(x) e^{-n \alpha_{n}(x)} e^{\mu x} \sum_{k=1}^{\infty} \frac{\left(n \alpha_{n}(x)\right)^{k-1}}{(k-1) !} f_{\mu}\left(\frac{k}{n}\right) \\
& -n \alpha_{n}^{\prime}(x) e^{-n \alpha_{n}(x)} e^{\mu x} \sum_{k=0}^{\infty} \frac{\left(n \alpha_{n}(x)\right)^{k}}{k !} f_{\mu}\left(\frac{k}{n}\right)-\mu e^{-n \alpha_{n}(x)} e^{\mu x} \sum_{k=0}^{\infty} \frac{\left(n \alpha_{n}(x)\right)^{k}}{k !} f_{\mu}\left(\frac{k}{n}\right) \\
= & n \alpha_{n}^{\prime}(x) e^{-n \alpha_{n}(x)} e^{\mu x} \sum_{k=0}^{\infty} \frac{\left(n \alpha_{n}(x)\right)^{k}}{k !}\left[f_{\mu}\left(\frac{k+1}{n}\right)-f_{\mu}\left(\frac{k}{n}\right)\right]=n \beta_{n} \mathcal{S}_{n}\left(\exp _{\mu} \Delta_{1 / n} f_{\mu} ; x\right)
\end{aligned}
$$

and

$$
\begin{aligned}
D_{\mu}^{2} \mathcal{S}_{n}(f ; x)= & n \mu \beta_{n} e^{-n \alpha_{n}(x)} e^{\mu x} \sum_{k=0}^{\infty} \frac{\left(n \alpha_{n}(x)\right)^{k}}{k !}\left[f_{\mu}\left(\frac{k+1}{n}\right)-f_{\mu}\left(\frac{k}{n}\right)\right] \\
& +n^{2} \beta_{n}^{2} e^{-n \alpha_{n}(x)} e^{\mu x} \sum_{k=1}^{\infty} \frac{\left(n \alpha_{n}(x)\right)^{k-1}}{(k-1) !}\left[f_{\mu}\left(\frac{k+1}{n}\right)-f_{\mu}\left(\frac{k}{n}\right)\right] \\
& -n^{2} \beta_{n}^{2} e^{-n \alpha_{n}(x)} e^{\mu x} \sum_{k=0}^{\infty} \frac{\left(n \alpha_{n}(x)\right)^{k}}{k !}\left[f_{\mu}\left(\frac{k+1}{n}\right)-f_{\mu}\left(\frac{k}{n}\right)\right] \\
& -n \mu \beta_{n} e^{-n \alpha_{n}(x)} e^{\mu x} \sum_{k=0}^{\infty} \frac{\left(n \alpha_{n}(x)\right)^{k}}{k !}\left[f_{\mu}\left(\frac{k+1}{n}\right)-f_{\mu}\left(\frac{k}{n}\right)\right] \\
= & n^{2} \beta_{n}^{2} e^{-n \alpha_{n}(x)} e^{\mu x} \sum_{k=0}^{\infty} \frac{\left(n \alpha_{n}(x)\right)^{k}}{k !}\left[f_{\mu}\left(\frac{k+2}{n}\right)-2 f_{\mu}\left(\frac{k+1}{n}\right)+f_{\mu}\left(\frac{k}{n}\right)\right] \\
= & n^{2} \beta_{n}^{2} \mathcal{S}_{n}\left(\exp _{\mu} \Delta_{1 / n}^{2} f ; x\right) .
\end{aligned}
$$

By induction, we have $D_{\mu}^{r} \mathcal{S}_{n}(f ; x)=n^{r} \beta_{n}^{r} \mathcal{S}_{n}\left(\exp _{\mu} \Delta_{1 / n}^{r} f_{\mu} ; x\right)$. 
$r$-th order $\mu$-derivative of the operator $\mathcal{K}_{n}$ is following.

Lemma 4. For every $r \in \mathbb{N}$ and $n \in \mathbb{N}$, we have

$$
D_{\mu}^{r} \mathcal{K}_{n}(f ; x)=n^{r+1} \beta_{n}^{r+1} \mathcal{S}_{n}\left(\exp _{\mu} \Delta_{1 / n}^{r+1} F_{\mu} ; x\right), \quad x \in \mathbb{R}^{+},
$$

where

$$
F_{\mu}(x)=\int_{0}^{x} f_{\mu}(t) d t
$$

Proof. We can write

$$
\begin{aligned}
\mathcal{K}_{n} f(x) & =n \beta_{n} e^{-n \alpha_{n}(x)} e^{\mu x} \sum_{k=0}^{\infty} \frac{\left(n \alpha_{n}(x)\right)^{k}}{k !} \int_{k / n}^{(k+1) / n} f_{\mu}(t) d t \\
& =n \beta_{n} e^{-n \alpha_{n}(x)} e^{\mu x} \sum_{k=0}^{\infty} \frac{\left(n \alpha_{n}(x)\right)^{k}}{k !}\left[F_{\mu}\left(\frac{k+1}{n}\right)-F_{\mu}\left(\frac{k}{n}\right)\right] .
\end{aligned}
$$

Using (4), we have

$$
\begin{aligned}
D_{\mu} \mathcal{K}_{n}(f ; x)= & \mu n \beta_{n} e^{-n \alpha_{n}(x)} e^{\mu x} \sum_{k=0}^{\infty} \frac{\left(n \alpha_{n}(x)\right)^{k}}{k !}\left[F_{\mu}\left(\frac{k+1}{n}\right)-F_{\mu}\left(\frac{k}{n}\right)\right] \\
& -n^{2} \beta_{n}^{2} e^{-n \alpha_{n}(x)} e^{\mu x} \sum_{k=0}^{\infty} \frac{\left(n \alpha_{n}(x)\right)^{k}}{k !}\left[F_{\mu}\left(\frac{k+1}{n}\right)-F_{\mu}\left(\frac{k}{n}\right)\right] \\
& +n^{2} \beta_{n}^{2} e^{-n \alpha_{n}(x)} e^{\mu x} \sum_{k=1}^{\infty} \frac{\left(n \alpha_{n}(x)\right)^{k-1}}{(k-1) !}\left[F_{\mu}\left(\frac{k+1}{n}\right)-F_{\mu}\left(\frac{k}{n}\right)\right] \\
& -\mu n \beta_{n} e^{-n \alpha_{n}(x)} e^{\mu x} \sum_{k=0}^{\infty} \frac{\left(n \alpha_{n}(x)\right)^{k}}{k !}\left[F_{\mu}\left(\frac{k+1}{n}\right)-F_{\mu}\left(\frac{k}{n}\right)\right] \\
= & n^{2} \beta_{n}^{2} e^{-n \alpha_{n}(x)} e^{\mu x} \sum_{k=0}^{\infty} \frac{\left(n \alpha_{n}(x)\right)^{k}}{k !}\left[F_{\mu}\left(\frac{k+2}{n}\right)-2 F_{\mu}\left(\frac{k+1}{n}\right)+F_{\mu}\left(\frac{k}{n}\right)\right] \\
= & n^{2} \beta_{n}^{2} e^{-n \alpha_{n}(x)} e^{\mu x} \sum_{k=0}^{\infty} \frac{\left(n \alpha_{n}(x)\right)^{k}}{k !}\left[\Delta_{1 / n}^{2} F_{\mu}\left(\frac{k}{n}\right)\right] \\
= & n^{2} \beta_{n}^{2} \mathcal{S}_{n}\left(\exp _{\mu} \Delta_{1 / n}^{2} F_{\mu} ; x\right) .
\end{aligned}
$$

By induction, we have $D_{\mu}^{r} \mathcal{K}_{n}(f ; x)=n^{r+1} \beta_{n}^{r+1} \mathcal{S}_{n}\left(\exp _{\mu} \Delta_{1 / n}^{r+1} F_{\mu} ; x\right)$.

The purpose of the following quantitative inequality with having the difference, if the approximation properties of one operator in the difference are known, it means the approximation properties of the other are known as well.

Let $f: \mathbb{R}^{+} \rightarrow \mathbb{R}$ be a continuous function. For $\delta>0$, the modulus of continuity of $f$ defined by

$$
\omega(f ; \delta)=\sup _{|t-x| \leq \delta}|f(t)-f(x)| .
$$

We will need following property of the modulus of continuity. For $f \in C\left(\mathbb{R}^{+}\right), \delta>0$ and $t, x \in \mathbb{R}^{+}$, the inequality

$$
|f(t)-f(x)| \leq \omega(f ;|t-x|)
$$

holds. 
Theorem 1. For any $r \in \mathbb{N}, n \in \mathbb{N}, x \in \mathbb{R}^{+}$and $f \in C^{(r)}\left(\mathbb{R}^{+}\right) \cap B\left(\mathbb{R}^{+}\right)$, the following inequality holds

$$
\left|D_{\mu}^{r} \mathcal{S}_{n}(f ; x)-\mathcal{S}_{n}\left(D_{\mu}^{r} f ; x\right)\right| \leq e^{\mu x}\left(\beta_{n}^{r} \omega\left(\exp _{\mu} D_{\mu}^{r} f ; r / n\right)+\left(\beta_{n}^{r}-1\right)\left\|D_{\mu}^{r} f\right\|_{\infty}\right) .
$$

Proof. Recall the equation (6),

$$
D_{\mu}^{r} \mathcal{S}_{n}(f ; x)=n^{r} \beta_{n}^{r} \mathcal{S}_{n}\left(\exp _{\mu} \Delta_{1 / n}^{r} f_{\mu} ; x\right) .
$$

Then

$$
\begin{aligned}
D_{\mu}^{r} \mathcal{S}_{n}(f ; x)-\mathcal{S}_{n}\left(D_{\mu}^{r} f ; x\right) & =n^{r} \beta_{n}^{r} \mathcal{S}_{n}\left(\exp _{\mu} \Delta_{1 / n}^{r} f_{\mu} ; x\right)-\beta_{n}^{r} \mathcal{S}_{n}\left(D_{\mu}^{r} f ; x\right)+\left(\beta_{n}^{r}-1\right) \mathcal{S}_{n}\left(D_{\mu}^{r} f ; x\right) \\
& =\beta_{n}^{r} \mathcal{S}_{n}\left(\exp _{\mu} n^{r} \Delta_{1 / n}^{r} f_{\mu}-D_{\mu}^{r} f_{\mu} ; x\right)+\left(\beta_{n}^{r}-1\right) \mathcal{S}_{n}\left(D_{\mu}^{r} f ; x\right)
\end{aligned}
$$

Using the equality

$$
n^{r} \Delta_{1 / n}^{r} f_{\mu}\left(\frac{k}{n}\right)=r !\left[\frac{k}{n}, \cdots, \frac{k+r}{n} ; f_{\mu}\right]=f_{\mu}^{(r)}\left(\xi_{k}\right),
$$

where $k / n \leq \xi_{k} \leq(k+r) / n$, we have

$$
D_{\mu}^{r} \mathcal{S}_{n}(f ; x)-\mathcal{S}_{n}\left(D_{\mu}^{r} f ; x\right)=\beta_{n}^{r} e^{-n \alpha_{n}(x)} e^{\mu x} \sum_{k=0}^{\infty} \frac{\left(n \alpha_{n}(x)\right)^{k}}{k !}\left[f_{\mu}^{(r)}\left(\xi_{k}\right)-e^{-\mu k / n} D_{\mu}^{r} f\left(\frac{k}{n}\right)\right] .
$$

Since $e^{\mu x} f_{\mu}^{(r)}(x)=D_{\mu}^{r} f(x)$, we have

$$
D_{\mu}^{r} \mathcal{S}_{n}(f ; x)-\mathcal{S}_{n}\left(D_{\mu}^{r} f ; x\right)=\beta_{n}^{r} \mathcal{S}_{n}\left(D_{\mu}^{r} f\left(\xi_{i}\right)-D_{\mu}^{r} f ; x\right)+\left(\beta_{n}^{r}-1\right) \mathcal{S}_{n}\left(D_{\mu}^{r} f ; x\right) .
$$

Finally, since $0 \leq \xi_{k}-k / n \leq r / n$, from (7) we have the estimate (8).

Now we give a similar result as in previous theorem for the operator $\mathcal{K}_{n}$.

Theorem 2. For any $n \in \mathbb{N}, x \in \mathbb{R}^{+}$and $f \in C\left(\mathbb{R}^{+}\right) \cap B\left(\mathbb{R}^{+}\right)$, the following inequality holds

$$
\left|D_{\mu}^{r} \mathcal{K}_{n}(f ; x)-\mathcal{K}_{n}\left(D_{\mu}^{r} f ; x\right)\right| \leq\left(\beta_{n}^{r+1}-\beta_{n}\right) e^{\mu x}\left\|f_{\mu}^{(r)}\right\|_{\infty}+\beta_{n} e^{\mu x} \omega\left(\exp _{\mu} D_{\mu}^{r} f ;(r+1) / n\right) .
$$

Proof. From Lemma 4, we can write

$$
D_{\mu}^{r} \mathcal{K}_{n}(f ; x)=n^{r+1} \beta_{n}^{r+1} \mathcal{S}_{n}\left(\exp _{\mu} \Delta_{1 / n}^{r+1} F_{\mu} ; x\right)=n^{r+1} \beta_{n}^{r+1} e^{-n \alpha_{n}(x)} e^{\mu x} \sum_{k=0}^{\infty} \frac{\left(n \alpha_{n}(x)\right)^{k}}{k !}\left[\Delta_{1 / n}^{r+1} F_{\mu}\left(\frac{k}{n}\right)\right] .
$$

Using the equality (9) for the function $F_{\mu}$, we can write

$$
\begin{aligned}
D_{\mu}^{r} \mathcal{K}_{n}(f ; x) & =\beta_{n}^{r+1} e^{-n \alpha_{n}(x)} e^{\mu x} \sum_{k=0}^{\infty} \frac{\left(n \alpha_{n}(x)\right)^{k}}{k !}(r+1) !\left[\frac{k}{n}, \ldots, \frac{k+r+1}{n} ; F_{\mu}\right] \\
& =\beta_{n}^{r+1} e^{-n \alpha_{n}(x)} e^{\mu x} \sum_{k=0}^{\infty} \frac{\left(n \alpha_{n}(x)\right)^{k}}{k !} F_{\mu}^{(r+1)}\left(\xi_{k}\right)=\beta_{n}^{r+1} e^{-n \alpha_{n}(x)} e^{\mu x} \sum_{k=0}^{\infty} \frac{\left(n \alpha_{n}(x)\right)^{k}}{k !} f_{\mu}^{(r)}\left(\xi_{k}\right),
\end{aligned}
$$

where $k / n \leq \xi_{k} \leq(k+r+1) / n$. From (5), we deduce

$$
\begin{aligned}
D_{\mu}^{r} \mathcal{K}_{n}(f ; x)-\mathcal{K}_{n}\left(D_{\mu}^{r} f ; x\right)= & \beta_{n}^{r+1} e^{-n \alpha_{n}(x)} e^{\mu x} \sum_{k=0}^{\infty} \frac{\left(n \alpha_{n}(x)\right)^{k}}{k !} f_{\mu}^{(r)}\left(\xi_{k}\right) \\
& -n \beta_{n} e^{-n \alpha_{n}(x)} e^{\mu x} \sum_{k=0}^{\infty} \frac{\left(n \alpha_{n}(x)\right)^{k}}{k !} \int_{k / n}^{(k+1) / n} e^{-\mu t} D_{\mu}^{r} f(t) d t .
\end{aligned}
$$


Using mean value theorem, we have

$$
\begin{aligned}
D_{\mu}^{r} \mathcal{K}_{n}(f ; x)-\mathcal{K}_{n}\left(D_{\mu}^{r} f ; x\right)= & \beta_{n}^{r+1} e^{-n \alpha_{n}(x)} e^{\mu x} \sum_{k=0}^{\infty} \frac{\left(n \alpha_{n}(x)\right)^{k}}{k !} f_{\mu}^{(r)}\left(\xi_{k}\right) \\
& -\beta_{n} e^{-n \alpha_{n}(x)} e^{\mu x} \sum_{k=0}^{\infty} \frac{\left(n \alpha_{n}(x)\right)^{k}}{k !} e^{-\mu \eta_{k}} D_{\mu}^{r} f\left(\eta_{k}\right),
\end{aligned}
$$

where $k / n \leq \eta_{k} \leq(k+1) / n$. Since $e^{\mu x} f_{\mu}^{(r)}(x)=D_{\mu}^{r} f(x)$, we deduce

$$
\begin{aligned}
D_{\mu}^{r} \mathcal{K}_{n}(f ; x)-\mathcal{K}_{n}\left(D_{\mu}^{r} f ; x\right)= & \left(\beta_{n}^{r+1}-\beta_{n}\right) e^{-n \alpha_{n}(x)} e^{\mu x} \sum_{k=0}^{\infty} \frac{\left(n \alpha_{n}(x)\right)^{k}}{k !} f_{\mu}^{(r)}\left(\xi_{k}\right) \\
& +\beta_{n} e^{-n \alpha_{n}(x)} e^{\mu x} \sum_{k=0}^{\infty} \frac{\left(n \alpha_{n}(x)\right)^{k}}{k !}\left[f_{\mu}^{(r)}\left(\xi_{k}\right)-e^{-\mu \eta_{k}} D_{\mu}^{r} f\left(\eta_{k}\right)\right] \\
= & \left(\beta_{n}^{r+1}-\beta_{n}\right) e^{-n \alpha_{n}(x)} e^{\mu x} \sum_{k=0}^{\infty} \frac{\left(n \alpha_{n}(x)\right)^{k}}{k !} f_{\mu}^{(r)}\left(\xi_{k}\right) \\
& +\beta_{n} e^{-n \alpha_{n}(x)} e^{\mu x} \sum_{k=0}^{\infty} \frac{\left(n \alpha_{n}(x)\right)^{k}}{k !}\left[e^{-\mu \xi_{k}} D_{\mu}^{r} f\left(\xi_{k}\right)-e^{-\mu \eta_{k}} D_{\mu}^{r} f\left(\eta_{k}\right)\right] .
\end{aligned}
$$

Thus, we have

$$
\left|D_{\mu}^{r} \mathcal{K}_{n}(f ; x)-\mathcal{K}_{n}\left(D_{\mu}^{r} f ; x\right)\right| \leq\left(\beta_{n}^{r+1}-\beta_{n}\right) e^{\mu x}\left\|f_{\mu}^{(r)}\right\|_{\infty}+\beta_{n} e^{\mu x} \omega\left(\exp _{\mu} D_{\mu}^{r} f ;(r+1) / n\right) .
$$

The following theorem provides an estimate of the difference between the operators $\mathcal{K}_{n}$ and $\mathcal{S}_{n}$.

Theorem 3. For any $n \in \mathbb{N}, x \in \mathbb{R}^{+}$and $f \in C\left(\mathbb{R}^{+}\right) \cap B\left(\mathbb{R}^{+}\right)$, the following inequality holds

$$
\left|\mathcal{K}_{n} f(x)-\mathcal{S}_{n} f(x)\right| \leq\left(\beta_{n}-1\right) e^{\mu x}\left\|f_{\mu}\right\|_{\infty}+\left(1+\frac{1}{\mu^{2}} e^{\mu x\left(e^{\mu / n}+2\right)}\right) \omega\left(f_{\mu} ; h\right),
$$

where

$$
h=\left[\frac{n}{2 \mu}\left(e^{2 \mu / n}-1\right)-\frac{2 n}{\mu}\left(e^{\mu / n}-1\right)+1\right] .
$$

Proof. Formulas (1) and (5) imply that

$$
\begin{aligned}
\mathcal{K}_{n} f(x)-\mathcal{S}_{n} f(x)= & \left(\alpha_{n}^{\prime}(x)-1\right) e^{-n \alpha_{n}(x)} e^{\mu x} \sum_{k=0}^{\infty} \frac{\left(n \alpha_{n}(x)\right)^{k}}{k !} \int_{k / n}^{(k+1) / n} f_{\mu}(t) d t \\
& +n e^{-n \alpha_{n}(x)} e^{\mu x} \sum_{k=0}^{\infty} \frac{\left(n \alpha_{n}(x)\right)^{k}}{k !} \int_{k / n}^{(k+1) / n}\left[f_{\mu}(t)-f_{\mu}\left(\frac{k}{n}\right)\right] d t .
\end{aligned}
$$

An easy consequence of the mean value theorem, we obtain

$$
\left|f_{\mu}(t)-f_{\mu}\left(\frac{k}{n}\right)\right| \leq\left(1+\frac{1}{h^{2}}\left(t-\frac{k}{n}\right)^{2}\right) \omega\left(f_{\mu} ; h\right) \leq\left(1+\frac{1}{\mu^{2} h^{2}}\left(e^{\mu t}-e^{\mu k / n}\right)^{2}\right) \omega\left(f_{\mu} ; h\right) .
$$


Then, by substituting above inequality, we have

$$
\begin{aligned}
\left|\mathcal{K}_{n} f(x)-\mathcal{S}_{n} f(x)\right| \leq & \left(\beta_{n}-1\right) e^{\mu x}\left\|f_{\mu}\right\|_{\infty} \\
& +\left(1+\frac{n}{\mu^{2} h^{2}} e^{-n \alpha_{n}(x)} e^{\mu x} \sum_{k=0}^{\infty} \frac{\left(n \alpha_{n}(x)\right)^{k}}{k !} \int_{k / n}^{(k+1) / n}\left(e^{\mu t}-e^{\mu k / n}\right)^{2} d t\right) \omega\left(f_{\mu} ; h\right) .
\end{aligned}
$$

Simple computations shows that

$$
n \int_{k / n}^{(k+1) / n}\left(e^{\mu t}-e^{\mu k / n}\right)^{2} d t=e^{2 \mu k / n}\left[\frac{n}{2 \mu}\left(e^{2 \mu / n}-1\right)-\frac{2 n}{\mu}\left(e^{\mu / n}-1\right)+1\right]
$$

and

$$
e^{-n \alpha_{n}(x)} e^{\mu x} \sum_{k=0}^{\infty} \frac{\left(n \alpha_{n}(x)\right)^{k}}{k !} e^{2 \mu k / n}=e^{\mu x\left(e^{\mu / n}+2\right)} .
$$

Taking into account the above relations, we get

$$
\begin{aligned}
\left|\mathcal{K}_{n} f(x)-\mathcal{S}_{n} f(x)\right| \leq & \left(\beta_{n}-1\right) e^{\mu x}\left\|f_{\mu}\right\|_{\infty} \\
& +\left(1+\frac{n}{\mu^{2} h^{2}} e^{-n \alpha_{n}(x)} e^{\mu x} \sum_{k=0}^{\infty} \frac{\left(n \alpha_{n}(x)\right)^{k}}{k !} \int_{k / n}^{(k+1) / n}\left(e^{\mu t}-e^{\mu k / n}\right)^{2} d t\right) \omega\left(f_{\mu} ; h\right) \\
\leq & \left(\beta_{n}-1\right)\left\|f_{\mu}\right\|_{\infty} \\
& +\left(1+\frac{1}{\mu^{2} h^{2}} e^{\mu x\left(e^{\mu / n}+2\right)}\left[\frac{n}{2 \mu}\left(e^{2 \mu / n}-1\right)-\frac{2 n}{\mu}\left(e^{\mu / n}-1\right)+1\right]\right) \omega\left(f_{\mu} ; h\right) .
\end{aligned}
$$

Setting

$$
h=\left[\frac{n}{2 \mu}\left(e^{2 \mu / n}-1\right)-\frac{2 n}{\mu}\left(e^{\mu / n}-1\right)+1\right]^{1 / 2}
$$

we have desired result.

In this section, we give a relation between the exponential Kantorovich operators $\mathcal{K}_{n}$ and Szàsz operators $\mathcal{S}_{n}$.

Theorem 4. For $f \in C^{(r)}\left(\mathbb{R}^{+}\right)$and $x \in \mathbb{R}^{+}$, we have

$$
\mathcal{K}_{n} f(x)=\sum_{j=0}^{r-1} \frac{1}{(j+1) ! n^{j}}\left(\mathcal{S}_{n} D_{\mu}^{(j)}(x)\right)+R_{n, r}(x),
$$

where

$$
R_{n, r}(x)=e^{-n \alpha_{n}(x)} e^{\mu x} \sum_{k=0}^{\infty} \frac{\left(n \alpha_{n}(x)\right)^{k}}{k !} \frac{1}{r !}\left[n \int_{k / n}^{(k+1) / n} \frac{D_{\mu}^{(r)} f\left(\xi_{t, x}\right)}{e^{\mu \xi_{t, x}}}\left(t-\frac{k}{n}\right)^{r} d t\right]
$$

is an absolute convergent series which is the remainder of the rth order, $u, x \in \mathbb{R}^{+}$and $\xi_{t, x} \in(t, x)$.

Proof. Using (4), we can write

$$
\left(\frac{f(x)}{e^{\mu x}}\right)^{(j)}=\frac{D_{\mu}^{(j)} f(x)}{e^{\mu x}}, \quad j \in \mathbb{N}
$$


For $f \in C^{(r)}\left(\mathbb{R}^{+}\right)$, we use the following version of the Taylor formula

$$
\begin{aligned}
\frac{f(t)}{e^{\mu t}} & =\sum_{j=0}^{r-1} \frac{1}{j !}\left(\frac{f(x)}{e^{\mu x}}\right)^{(j)}(t-x)^{j}+\frac{1}{r !}\left(\frac{f}{\exp _{\mu}}\right)^{(r)}\left(\xi_{t, x}\right)(t-x)^{r} \\
& =\sum_{j=0}^{r-1} \frac{1}{j !} \frac{D_{\mu}^{(j)} f(x)}{e^{\mu x}}(t-x)^{j}+\frac{1}{r !} \frac{D_{\mu}^{(r)} f\left(\xi_{t, x}\right)}{e^{\mu \xi_{t, x}}}(t-x)^{r},
\end{aligned}
$$

where $t, x \in \mathbb{R}^{+}$and $\xi_{t, x} \in(t, x)$. Taking $x=\frac{k}{n}$, and simple computations lead to

$$
\begin{aligned}
n \int_{k / n}^{(k+1) / n} e^{-\mu t} f(t) d t & =\sum_{j=0}^{r-1} \frac{1}{j !} \frac{D_{\mu}^{(j)} f\left(\frac{k}{n}\right)}{e^{\mu \frac{k}{n}}} n \int_{\frac{k}{n}}^{\frac{k+1}{n}}\left(t-\frac{k}{n}\right)^{j} d t+\frac{n}{r !} \int_{k / n}^{(k+1) / n} \frac{D_{\mu}^{(r)} f\left(\xi_{t, x}\right)}{e^{\mu \xi_{t, x}}}\left(t-\frac{k}{n}\right)^{r} d t \\
& =\sum_{j=0}^{r-1} \frac{1}{(j+1) !} \frac{D_{\mu}^{(j)} f(k / n)}{e^{\mu \frac{k}{n}}} \frac{1}{n^{j}}+\frac{n}{r !} \int_{k / n}^{(k+1) / n} \frac{D_{\mu}^{(j)} f\left(\xi_{t, x}\right)}{e^{\mu \xi_{t, x}}}\left(t-\frac{k}{n}\right)^{r} d t
\end{aligned}
$$

for every $n \geq 1$.

Thus from (5), it follows that

$$
\begin{aligned}
\mathcal{K}_{n} f(x)= & \alpha_{n}^{\prime}(x) e^{-n \alpha_{n}(x)} e^{\mu x} \sum_{k=0}^{\infty} \frac{\left(n \alpha_{n}(x)\right)^{k}}{k !}\left[\sum_{j=0}^{r-1} \frac{1}{(j+1) !} \frac{D_{\mu}^{(j)} f\left(\frac{k}{n}\right)}{e^{\mu \frac{k}{n}}} \frac{1}{n^{j}}\right. \\
& +\frac{n}{r !} \int_{k / n}^{(k+1) / n} \frac{D_{\mu}^{(j)} f(\xi t, x)}{\left.e^{\mu \xi_{t, x}}\left(t-\frac{k}{n}\right)^{r} d t\right]} \\
= & \alpha_{n}^{\prime}(x) \sum_{j=0}^{r-1} \frac{e^{\mu x} e^{-n \alpha_{n}(x)}}{(j+1) ! n^{j}} \sum_{k=0}^{\infty} \frac{\left(n \alpha_{n}(x)\right)^{k}}{k !} \frac{D_{\mu}^{(j)} f(k / n)}{e^{\mu k / n}} \\
& +\alpha_{n}^{\prime}(x) e^{-n \alpha_{n}(x)} e^{\mu x} \sum_{k=0}^{\infty} \frac{\left(n \alpha_{n}(x)\right)^{k}}{k !} \frac{1}{r !}\left[n \int_{k / n}^{(k+1) / n} \frac{D_{\mu}^{(r)} f\left(\xi_{t, x}\right)}{e^{\mu \xi_{t, x}}}\left(t-\frac{k}{n}\right)^{r} d t\right] \\
= & \sum_{j=0}^{r-1} \frac{\alpha_{n}^{\prime}(x)}{(j+1) ! n^{j}}\left(\mathcal{S}_{n} D_{\mu}^{(j)} f(x)\right)+R_{n, r}(x) .
\end{aligned}
$$

Also $R_{n, r}(x)$ is an absolutely convergent series.

\section{Convergence in variation}

In this section we establish variation detracting property and convergence in variation of Szàsz operators $\mathcal{S}_{n}$ and Szàsz-Kantorovich operators $\mathcal{K}_{n}$ in the space of functions of bounded variation.

Let us start by recalling the variation detracting property and convergence in variation of a sequence of operators.

Let $I \subset \mathbb{R}$ be a finite or infinite interval. In this section, $V_{I}[f]$ represents the total Jordan variation of the real-valued function $f$ defined on $I$ and $T V[I]$ is the space of all functions of bounded variation on $I$ with the seminorm $\|f\|_{T V}:=V_{[I]}[f]$, where $f \in A C[I]$, which is the space of absolutely continuous functions. $A C[I]$ is a closed subspace of $T V[I]$. Also, $B V(I)$ represents the class of all functions of bounded variation on $I$ with the norm

$$
\|f\|_{B V(I)}:=V_{I}[f]+|f(0)|, \quad f \in B V(I) .
$$


In order to obtain the variation detracting property of a given linear operator $L$ defined on $B V(I)$, the operator $L$ has the property $V_{I}[L f] \leq V_{I}[f]$, for each $f \in B V(I)$.

Let $\left(L_{n}\right)$ be a sequence of linear operator defined on $B V(I)$. For a given $f \in B V(I)$, if the following statement holds

$$
\lim _{n \rightarrow \infty} V_{I}\left[L_{n} f-f\right]=0,
$$

then the sequence $\left(L_{n}\right)_{n \geq 1}$ converges in variation to $f$.

In addition, if $f \in A C(I)$, the space of all absolutely continuous real-valued functions defined on $I$, then $f^{\prime}$ exists a.e. on $I$. Hence $V_{I}[f]$ can be calculated by

$$
V_{I}[f]=\int_{I}\left|f^{\prime}(t)\right| d t
$$

in $L_{1}(I)$, the space of all real-valued Lebesque integrable functions defined on $I$.

If $\lim _{n \rightarrow \infty} V_{I}\left[\varphi_{n}-f\right]=0$ for a sequence $\left(\varphi_{n}\right)_{n \geq 1}$ in $A C(I)$, then also $f \in A C(I)$ and

$$
V_{I}\left[\varphi_{n}-f\right]=\int_{I}\left|\varphi_{n}^{\prime}(t)-f^{\prime}(t)\right| d t .
$$

As a result, for a given $\varphi_{n} \in A C(I)$ the convergence in variation of $\left(\varphi_{n}\right)_{n \geq 1}$ to $f$ means the convergences of $\left(\varphi_{n}^{\prime}\right)$ to $f^{\prime}$ in the $L_{1}(I)$-norm. More details can be found in $[7,13]$.

First, we show that the operator $\mathcal{S}_{n}$ is bounded operator with respect to $B V$-norm.

Theorem 5. If $f \in T V[0, \infty)$, we have

$$
\left\|\frac{\mathcal{S}_{n} f}{\exp _{\mu}}\right\|_{B V} \leq\left\|\frac{f}{\exp _{\mu}}\right\|_{B V}
$$

Proof. By simple computations we can write

$$
\left(\frac{\mathcal{S}_{n} f}{\exp _{\mu}}\right)^{\prime}(x)=n \beta_{n} e^{-n \alpha_{n}(x)} \sum_{k=0}^{\infty}\left[\left(\frac{f}{\exp _{\mu}}\right)\left(\frac{k+1}{n}\right)-\left(\frac{f}{\exp _{\mu}}\right)\left(\frac{k}{n}\right)\right] \frac{\left(n \alpha_{n}(x)\right)^{k}}{k !} .
$$

Then, we have

$$
\begin{aligned}
V_{[0, \infty)}\left[\frac{\mathcal{S}_{n} f}{\exp _{\mu}}\right] & =\int_{0}^{\infty}\left|\left(\frac{\mathcal{S}_{n} f}{\exp _{\mu}}\right)^{\prime}(x)\right| d x \\
& \leq \sum_{k=0}^{\infty}\left|\left(\frac{f}{\exp _{\mu}}\right)\left(\frac{k+1}{n}\right)-\left(\frac{f}{\exp _{\mu}}\right)\left(\frac{k}{n}\right)\right| n \int_{0}^{\infty} e^{-n \alpha_{n}(x)} \frac{\left(n \alpha_{n}(x)\right)^{k}}{k !} \beta_{n} d x .
\end{aligned}
$$

Since

$$
n \int_{0}^{\infty} e^{-n \alpha_{n}(x)} \frac{\left(n \alpha_{n}(x)\right)^{k}}{k !} \beta_{n} d x=\frac{\Gamma(k+1)}{k !}=1,
$$

we have

$$
V_{[0, \infty)}\left[\frac{\mathcal{S}_{n} f}{\exp _{\mu}}\right] \leq \sum_{k=0}^{\infty}\left|\left(\frac{f}{\exp _{\mu}}\right)\left(\frac{k+1}{n}\right)-\left(\frac{f}{\exp _{\mu}}\right)\left(\frac{k}{n}\right)\right| \leq V_{[0, \infty)}\left[\frac{f}{\exp _{\mu}}\right] .
$$

Considering the above inequality, we have

$$
\left\|\frac{\mathcal{S}_{n} f}{\exp _{\mu}}\right\|_{B V}=V_{[0, \infty)}\left[\frac{\mathcal{S}_{n} f}{\exp _{\mu}}\right]+\left|\mathcal{S}_{n} f(0)\right| \leq V_{[0, \infty)}\left[\frac{f}{\exp _{\mu}}\right]+|f(0)| \leq\left\|\frac{f}{\exp _{\mu}}\right\|_{B V} .
$$


Theorem 6. If $f \in T V[0, \infty)$, then we have

$$
V_{[0, \infty)}\left[\frac{\mathcal{K}_{n} f}{\exp _{\mu}}\right] \leq V_{[0, \infty)}\left[\frac{f}{\exp _{\mu}}\right] \text { and } \quad\left\|\frac{\mathcal{K}_{n} f}{\exp _{\mu}}\right\|_{T V} \leq\left\|\frac{f}{\exp _{\mu}}\right\|_{T V} .
$$

Proof. Considering the equality

$$
F_{k, n}:=\int_{k / n}^{(k+1) / n} f_{\mu}(t) d t=\int_{0}^{1} f_{\mu}\left(\frac{k+u}{n}\right) d u,
$$

we can write

$$
\left(\frac{\mathcal{K}_{n} f}{\exp _{\mu}}\right)^{\prime}(x)=\alpha_{n}^{\prime \prime}(x) e^{-n \alpha_{n}(x)} \sum_{k=0}^{\infty} \frac{\left(n \alpha_{n}(x)\right)^{k}}{k !} F_{k, n}+n\left(\alpha_{n}^{\prime}(x)\right)^{2} e^{-n \alpha_{n}(x)} \sum_{k=0}^{\infty}\left[F_{k+1, n}-F_{k, n}\right] \frac{\left(n \alpha_{n}(x)\right)^{k}}{k !},
$$

where $\alpha_{n}^{\prime \prime}(x)=0$. Then

$$
\begin{aligned}
\left(\frac{\mathcal{K}_{n} f}{\exp _{\mu}}\right)^{\prime}(x) & =n\left(\alpha_{n}^{\prime}(x)\right)^{2} e^{-n \alpha_{n}(x)} \sum_{k=0}^{\infty}\left[F_{k+1, n}-F_{k, n}\right] \frac{\left(n \alpha_{n}(x)\right)^{k}}{k !} \\
& =n \alpha_{n}^{\prime}(x) e^{-n \alpha_{n}(x)} \sum_{k=0}^{\infty}\left[F_{k+1, n}-F_{k, n}\right] \frac{\left(n \alpha_{n}(x)\right)^{k}}{k !} \alpha_{n}^{\prime}(x) \\
& =\beta_{n} e^{-n \alpha_{n}(x)} n \sum_{k=0}^{\infty}\left[F_{k+1, n}-F_{k, n}\right] \frac{\left(n \alpha_{n}(x)\right)^{k}}{k !} \alpha_{n}^{\prime}(x) .
\end{aligned}
$$

Therefore

$$
\begin{aligned}
V_{[0, \infty)}\left[\frac{\mathcal{K}_{n} f}{\exp _{\mu}}\right] & =\int_{0}^{\infty}\left|\left(\frac{\mathcal{K}_{n} f}{\exp _{\mu}}\right)^{\prime}(x)\right| d x \\
& =\int_{0}^{\infty}\left|\frac{\mu}{n\left(e^{\mu / n}-1\right)} \sum_{k=0}^{\infty}\left[F_{k+1, n}-F_{k, n}\right] \frac{\left(n \alpha_{n}(x)\right)^{k}}{k !} n e^{-n \alpha_{n}(x)} \alpha_{n}^{\prime}(x)\right| d x \\
& \leq \frac{\mu}{n\left(e^{\mu / n}-1\right)} \sum_{k=0}^{\infty}\left|F_{k+1, n}-F_{k, n}\right| \frac{n^{k}}{k !} \int_{0}^{\infty} n e^{-n \alpha_{n}(x)}\left(\alpha_{n}(x)\right)^{k} \alpha_{n}^{\prime}(x) d x
\end{aligned}
$$

Since

$$
\frac{n^{k}}{k !} \int_{0}^{\infty} n e^{-n \alpha_{n}(x)}\left(\alpha_{n}(x)\right)^{k} \alpha_{n}^{\prime}(x) d x=\frac{\Gamma(k+1)}{k !}=1
$$

then we have

$$
V_{[0, \infty)}\left[\frac{\mathcal{K}_{n} f}{\exp _{\mu}}\right] \leq \frac{\mu}{n\left(e^{\mu / n}-1\right)} \sum_{k=0}^{\infty}\left|F_{k+1, n}-F_{k, n}\right|
$$

and considering $\mu \leq n\left(e^{\mu / n}-1\right)$, we can write

$$
V_{[0, \infty)}\left[\frac{\mathcal{K}_{n} f}{\exp _{\mu}}\right] \leq \sum_{k=0}^{\infty}\left|F_{k+1, n}-F_{k, n}\right|
$$

Since

$$
\sum_{k=0}^{\infty}\left|F_{k+1, n}-F_{k, n}\right| \leq \int_{0}^{1} \sum_{k=0}^{\infty}\left|f_{\mu}\left(\frac{k+1+u}{n}\right)-f_{\mu}\left(\frac{k+u}{n}\right)\right| d u \leq V_{[0, \infty)}\left[\frac{f}{\exp _{\mu}}\right]=\|f\|_{T V},
$$


then we have

$$
V_{[0, \infty)}\left[\frac{\mathcal{K}_{n} f}{\exp _{\mu}}\right] \leq V_{[0, \infty)}\left[\frac{f}{\exp _{\mu}}\right]
$$

That is

$$
\left\|\frac{\mathcal{K}_{n} f}{\exp _{\mu}}\right\|_{T V} \leq\left\|\frac{f}{\exp _{\mu}}\right\|_{T V}
$$

This completes the proof.

Theorem 7. If $f \in T V\left(\mathbb{R}^{+}\right)$, then we have

$$
\lim _{n \rightarrow \infty}\left\|\frac{\mathcal{S}_{n} f}{\exp _{\mu}}-\frac{f}{\exp _{\mu}}\right\|_{T V}=0
$$

Furthermore in addition, if (11) holds for $f \in C\left(\mathbb{R}^{+}\right)$, then $f \in A C\left(\mathbb{R}^{+}\right)$.

Proof. We have

$$
\begin{aligned}
\left(\frac{\mathcal{S}_{n} f}{\exp _{\mu}}\right)^{\prime}(x) & =n \beta_{n} e^{-n \alpha_{n}(x)} \sum_{k=0}^{\infty} \frac{\left(n \alpha_{n}(x)\right)^{k}}{k !}\left[f_{\mu}\left(\frac{k+1}{n}\right)-f_{\mu}\left(\frac{k}{n}\right)\right] \\
& =n \beta_{n} e^{-n \alpha_{n}(x)} \sum_{k=0}^{\infty} \frac{\left(n \alpha_{n}(x)\right)^{k}}{k !} \int_{k / n}^{(k+1) / n} f_{\mu}^{\prime}(t) d t=\left(\frac{\mathcal{K}_{n}}{\exp _{\mu}}\right)\left(D_{\mu} f\right)(x)
\end{aligned}
$$

and

$$
\begin{aligned}
V_{\mathbb{R}^{+}}\left[\frac{\mathcal{S}_{n} f}{\exp _{\mu}}-\frac{f}{\exp _{\mu}}\right] & =\int_{0}^{\infty}\left|\left(\frac{S_{n} f}{\exp _{\mu}}\right)^{\prime}(x)-\left(\frac{f}{\exp _{\mu}}\right)^{\prime}(x)\right| d x \\
& =\int_{0}^{\infty}\left|\left(\frac{\mathcal{K}_{n}}{\exp _{\mu}}\right)\left(D_{\mu} f\right)(x)-\left(\frac{D_{\mu} f}{\exp _{\mu}}\right)(x)\right| d x
\end{aligned}
$$

Since

$$
\left(\frac{\mathcal{S}_{n} f}{\exp _{\mu}}\right)^{\prime}(x)=\left(\frac{\mathcal{K}_{n}}{\exp _{\mu}}\right)\left(D_{\mu} f\right)(x)
$$

and $\mathcal{K}_{n} f$ is continuously differentiable of order $r$ on $\mathbb{R}^{+}$from Lemma 4, considering Orlicz's results [20, Theorem 2.1.2], to unbounded interval by [13, p.315], and taking into account (10) we have

$$
V_{\mathbb{R}^{+}}\left[\frac{\mathcal{S}_{n} f}{\exp _{\mu}}-\frac{f}{\exp _{\mu}}\right]=\int_{0}^{\infty}\left|\left(\frac{\mathcal{K}_{n} f^{\prime}}{\exp _{\mu}}\right)(x)-\left(\frac{f^{\prime}}{\exp _{\mu}}\right)(x)\right| d x \rightarrow 0, \quad n \rightarrow \infty,
$$

in the norm of $L_{1}\left(\mathbb{R}^{+}\right)$, the Banach space of all real-valued Lebesgue integrable functions defined on $\mathbb{R}^{+}$. Therefore, we obtain

$$
\lim _{n \rightarrow \infty} V_{\mathbb{R}^{+}}\left[\frac{\mathcal{S}_{n} f}{\exp _{\mu}}-\frac{f}{\exp _{\mu}}\right]=0
$$

Conversely, the condition $f \in A C\left(\mathbb{R}^{+}\right)$is also necessary for the convergence in variation, since $\mathcal{S}_{n} f \in A C\left(\mathbb{R}^{+}\right)$yields $f \in A C\left(\mathbb{R}^{+}\right)$. Because of $A C\left(\mathbb{R}^{+}\right)$is a closed subspace of $B V\left(\mathbb{R}^{+}\right)$in the seminorm $V_{\mathbb{R}^{+}}[\cdot]$. This completes the proof. 


\section{Numerical results}

In this section, we will give some graphical results supporting the related theorems using the Mathematica programme.

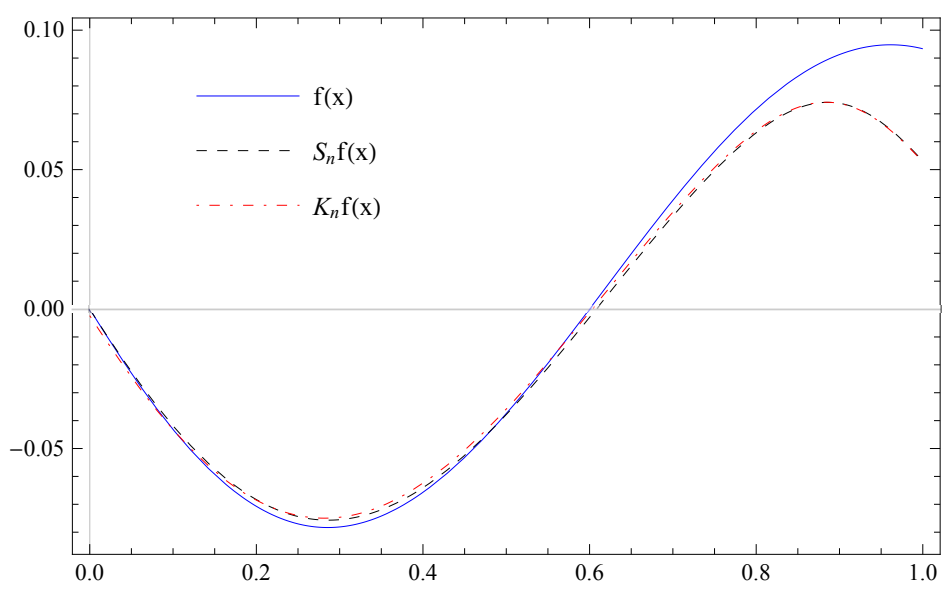

Figure 1. The plots of functions separately

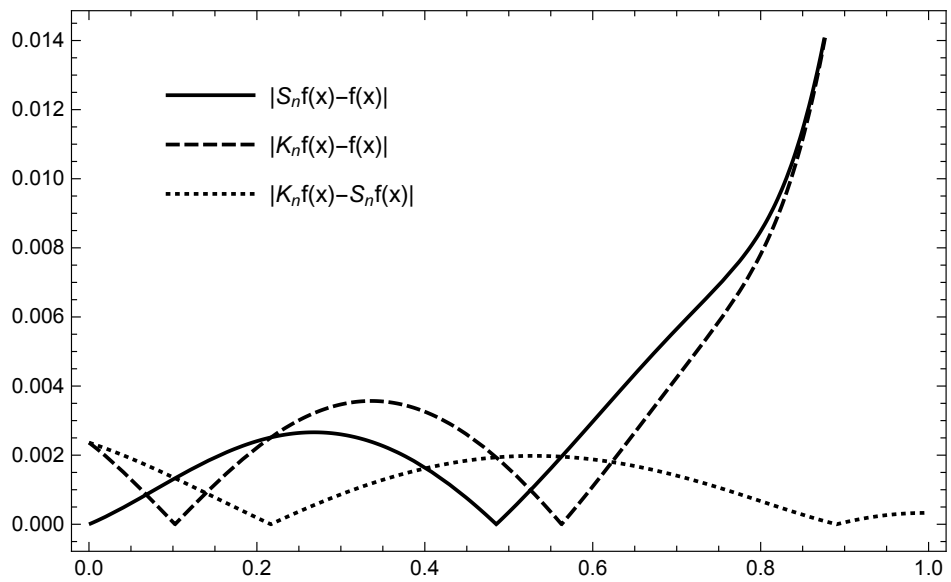

Figure 2. The plots of differences between operators

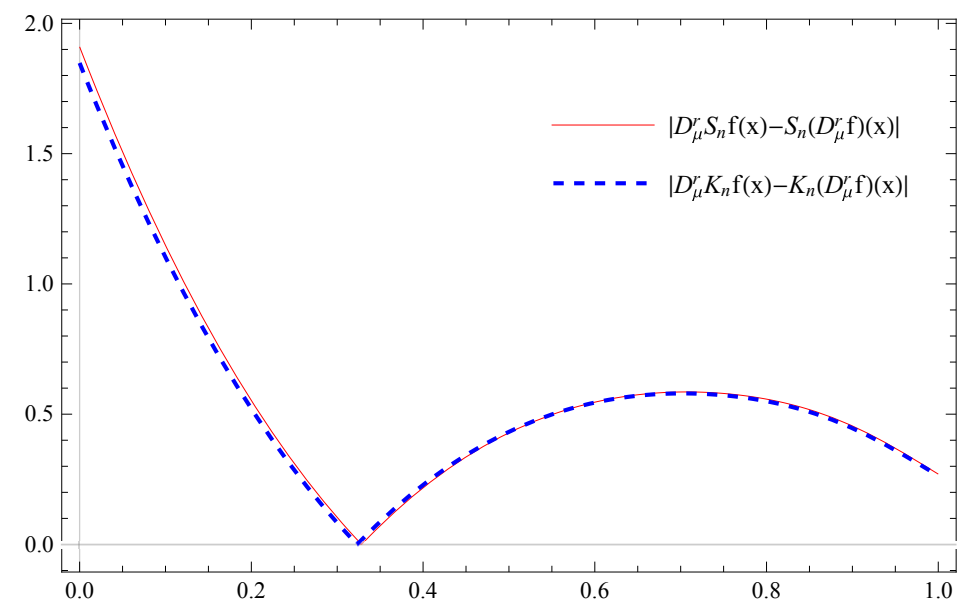

Figure 3. The plot of quantitative estimates for higher order $\mu$-derivatives

Example 1. Let

$$
f(x)=x^{5}-\frac{91}{30} x^{4}+\frac{349}{150} x^{3}+\frac{7}{25} x^{2}-\frac{12}{25} x, \quad \mu=1, \quad r=3, \quad \text { and } \quad n=100 .
$$


In Figure 1, the graphs of the function $f(x)$ and the graphs of the operators $\mathcal{S}_{n} f$ and $\mathcal{K}_{n} f$ are given. In Figure 2, some absolute values of the differences $\left|\mathcal{S}_{n} f(x)-f(x)\right|$, $\left|\mathcal{K}_{n} f(x)-f(x)\right|$, and $\left|\mathcal{K}_{n} f(x)-\mathcal{S}_{n} f(x)\right|$ are compared as graphical. In Figure 3 , the graphs of the some absolute values of the differences higher order $\mu$-derivatives of the Szàsz operators and the Kantorovich operators acting on the same order $\mu$-derivative of the function $f(x)$ are given (i.e. $\left|D_{\mu}^{r} \mathcal{S}_{n}(f ; x)-\mathcal{S}_{n}\left(D_{\mu}^{r} f ; x\right)\right|$ and $\left.\left|D_{\mu}^{r} \mathcal{K}_{n}(f ; x)-\mathcal{K}_{n}\left(D_{\mu}^{r} f ; x\right)\right|\right)$.

Example 2. Let

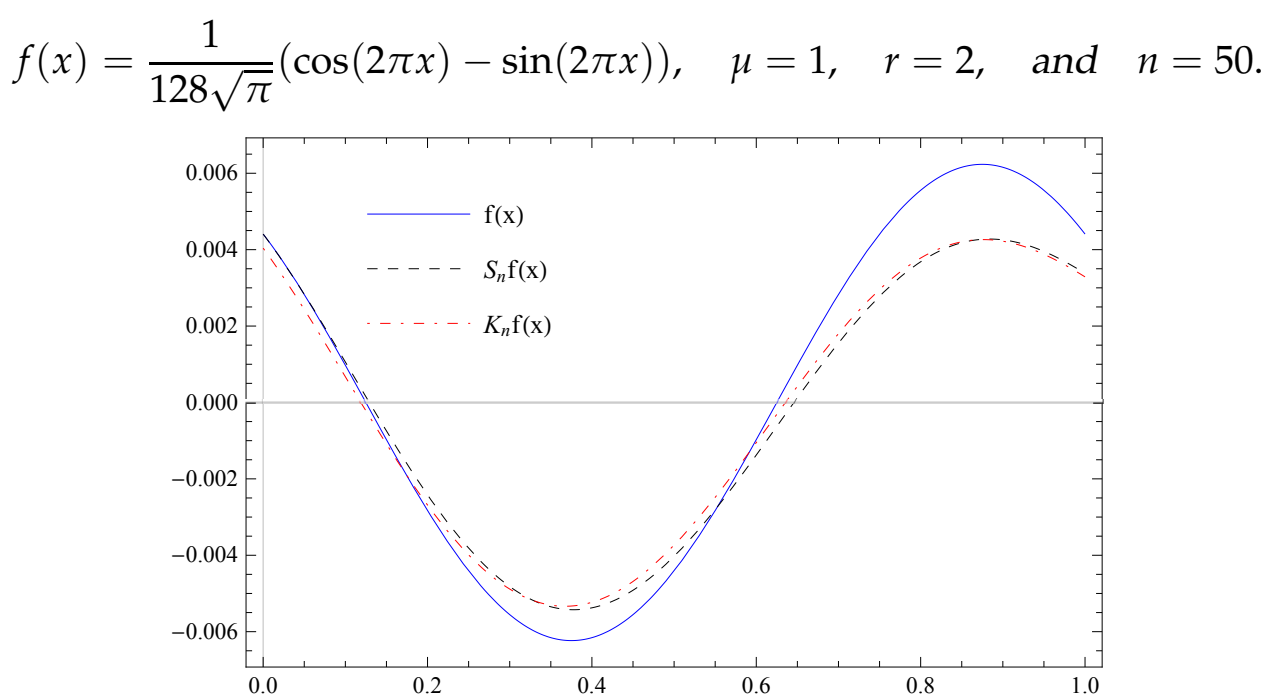

Figure 4. The plots of functions separately according to Example 2

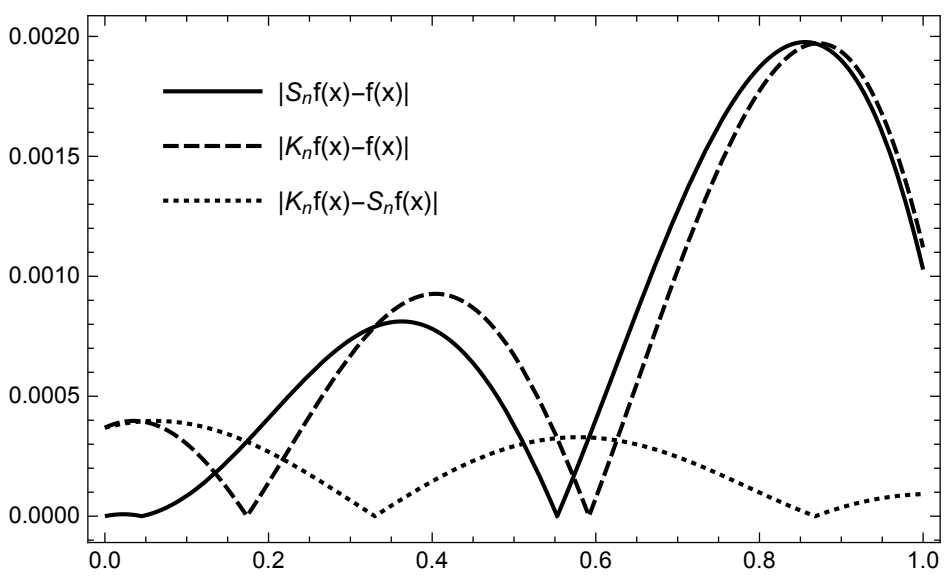

Figure 5. The plots of differences between operators according to Example 2

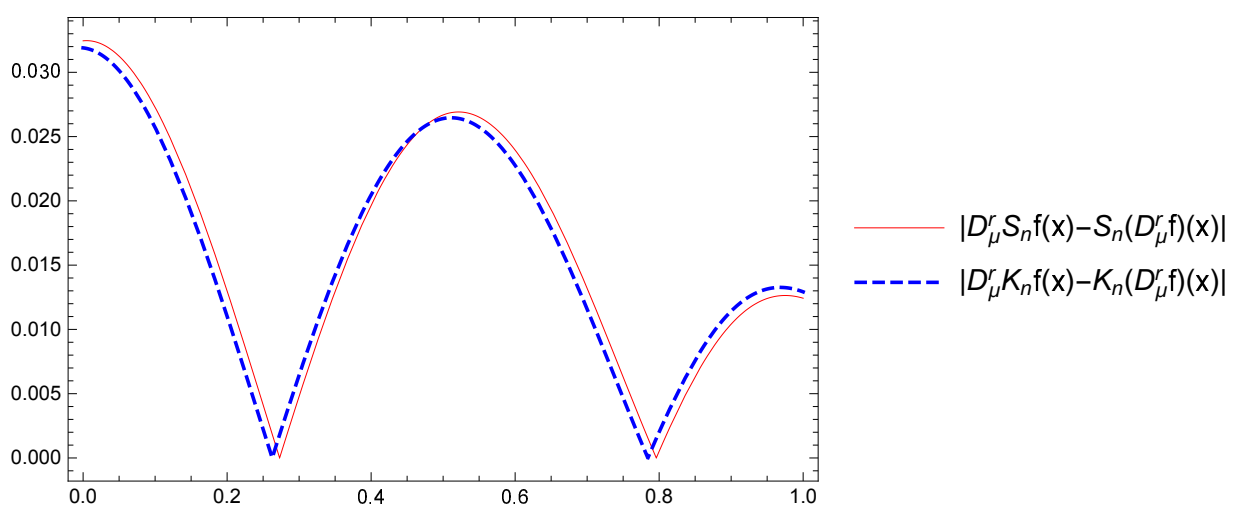

Figure 6. The plot of quantitative estimates for higher order $\mu$-derivatives according to Example 2 
In Figure 4, the graphs of the function $f(x)$ and the graphs of the operators $\mathcal{S}_{n} f$ and $\mathcal{K}_{n} f$ are given. In Figure 5, some absolute values of the differences $\left|\mathcal{S}_{n} f(x)-f(x)\right|$, $\left|\mathcal{K}_{n} f(x)-f(x)\right|$, and $\left|\mathcal{K}_{n} f(x)-\mathcal{S}_{n} f(x)\right|$ are compared as graphical. Finally, in Figure 6, the graphs of the some absolute values of the differences higher order $\mu$-derivatives of the Szàsz operators and the Kantorovich operators acting on the same order $\mu$-derivative of the function $f(x)$ (i.e. $\left|D_{\mu}^{r} \mathcal{S}_{n}(f ; x)-\mathcal{S}_{n}\left(D_{\mu}^{r} f ; x\right)\right|$ and $\left.\left|D_{\mu}^{r} \mathcal{K}_{n}(f ; x)-\mathcal{K}_{n}\left(D_{\mu}^{r} f ; x\right)\right|\right)$.

\section{References}

[1] Abel U., Agratini O. On the variation detracting property of operators of Balázs and Szabados. Acta Math. Hungar. 2016, 150 (2), 383-395. doi:10.1007/s10474-016-0642-x

[2] Acar T., Aral A., Cárdenas-Morales D., Garrancho P. Szász-Mirakyan type operators which fix exponentials. Results Math. 2017, 72 (3), 1393-1404. doi:10.1007/s00025-017-0665-9

[3] Acar T., Aral A., Gonska H.On Szász-Mirakyan operators preserving e $e^{2 a x}, a>0$. Mediterr. J. Math. 2017,14 (1), 6. doi:10.1007/s00009-016-0804-7

[4] Acu A.M., Aral A., Raşa I. Generalized Bernstein Kantorovich operators: Voronovskaya type results, convergence in variation. Carpathian J. Math. 2022, 38 (1), 1-12. doi:10.37193/cjm.2022.01.01

[5] Acu A.M., Raşa I. New estimates for the differences of positive linear operators. Numer. Algorithm 2016, 73 (3), 775-789. doi:10.1007/s11075-016-0117-8

[6] Acu A.M., Raşa I. Estimates for the differences of positive linear operators and their derivatives. Numer. Algorithms 2020, 85 (2), 191-208. doi;10.1007/s11075-019-00809-4

[7] Agratini O. On the variation detracting property of a class of operators. Appl. Math. Lett. 2006, 19, 1261-1264. doi:10.1016/j.aml.2005.12.007

[8] Aral A., Cárdenas-Morales D., Garrancho P. Bernstein-type operators that reproduce exponential functions. J. Math. Inequal. 2018, 12 (3), 861-872. doi:10.7153/jmi-2018-12-64

[9] Aral A., Erbay H. A note on the difference of positive operators and numerical aspects. Mediterr. J. Math. 2020, 17 (2), 45. doi:10.1007/s00009-020-1489-5

[10] Aral A., Otrocol D., Raşa I. On approximation by some Bernstein Kantorovich exponential-type polynomials. Period. Math. Hungar. 2019, 79 (2), 236-254. doi:10.1007/s10998-019-00284-3

[11] Aral A., Limmam L.M., Ozsarac F. Approximation properties of Szász-Mirakyan-Kantorovich type operators. Math. Methods Appl. Sci. 2019, 42 (16), 5233-5240. doi:10.1002/mma.5280

[12] Deniz E., Aral A., Gupta V. Note on Szász-Mirakyan-Durrmeyer operators preserving $e^{2 a x}, a>0$. Numer. Funct. Anal. Optim. 2017, 39 (2), 201-207. doi:10.1080/01630563.2017.1358179

[13] Bardaro C., Butzer P.L., Stens R.L., Vinti G. Convergence in variation and rates of approximation for Bernstein-type polynomials and singular convolution integrals. Analysis (Berlin) 2003, 23 (4), 299-346. doi: 10.1524/anly.2003.23.4.299

[14] Gonska H., Pitul P., Raşa I. On differences of positive linear operators. Carpathian J. Math. 2006, 22 (1-2), 65-78.

[15] Gonska H., Pitul P., Raşa I. On Peano's form of the Taylor remainder, Voronovskaja's theorem and the commutator of positive linear operators. In: Agratini O., Blaga P. (Eds.) Proc. Int. Conf. “Numerical Analysis and Approximation Theory", Cluj-Napoca, Romania, July 5-8, 2006. Casa Cărtiide Stiintă, Cluj-Napoca, 2006, 55-80.

[16] Gonska H., Raşa I. Differences of positive linear operators and the second order modulus. Carpathian J. Math. 2008, 24 (3), 332-340.

[17] Gupta V., Tachev G. On approximation properties of Phillips operators preserving exponential functions. Mediterr. J. Math. 2017, 14 (4), 177. doi:10.1007/s00009-017-0981-z 
[18] Gupta V., López-Moreno A.-J. Phillips operators preserving arbitrary exponential functions, $e^{a x}, e^{b x}$. Filomat 2018, 32 (14), 5071-5082. doi:10.2298/FIL1814071G

[19] Karsli H. On convergence of Chlodovsky and Chlodovsky-Kantorovich polynomials in the variation seminorm. Mediterr. J. Math. 2013, 10 (1), 41-56. doi:10.1007/s00009-012-0186-4

[20] Lorentz G.G. Bernstein Polynomials. University of Toronto Press, Toronto, 1953.

[21] Lupaş A. The approximation by means of some linear positive operators. In: Müller M.W., Felten M., Mache D.H. (Eds.) Approximation Theory, 86. Akademie Verlag, Berlin, 1995, 201-227.

[22] Păltănea R. A note on Bernstein Kantorovich operators. Bull. Transilv. Univ. Braşov Ser. III 2013, 6(55) (2), 27-32.

Бозкурт К., Аіммам М.А., Арал А. Узагальнення операторів Саса: кількісна оцінка та обмежена варіація // Карпатські матем. публ. - 2021. - Т.13, №3. - С. 775-789.

Отримано оцінки різниці операторів експоненційного типу Саса та Саса-Канторовича. Подібні оцінки наведено і для $\mu$-похідних вищого порядку операторів Саса та операторів типу Саса-Канторовича, що діють на $\mu$-похідні функцій того ж порядку. Ці оцінки наведено в кількісній формі з використанням першого модуля неперервності. Отримано збіжність за варіацією операторів у просторі функцій з обмеженою варіацією відносно варіаційної напівнорми. Отримані результати визначають загальну структуру, що охоплює що охоплює відомі результати у літературі.

Ключові слова і фрази: експоненщійний оператор Саса, експоненційний оператор Саса-Канторовича, збіжність за варіацією. 